All intersector sequences of order three will be closed if the invariant

$$
p^{2}\left(p_{21}^{2} \alpha^{2}+p_{12}^{2} \beta^{2}\right)+2 p_{12}^{3} p_{21}^{3} \alpha^{2} \beta^{2}
$$

vanishes. This can happen either if $p=0, \alpha=0$ (or $\beta=0$ ), or $\alpha=0, \beta=0$. In the first case one branch of the flecnode curve of $R_{y z}$ is plane, $(\alpha=0)$, and $R_{\psi \phi}$ degenerates into the tangents of a plane curve. In the second case both branches of the flecnode curve of $R_{y z}$ are plane and $R_{\psi \phi}$ degenerates into a straight line.

It is obvious that in the preceding developments the order of the lines $l_{y z}, l_{\psi \phi}, l_{\eta \theta}$ can be reversed without in any way affecting results. The analysis would be based upon a system of firstorder equations of the same type as (4), (5) and obtainable from (4), (5) by simple processes.

The University of Washington

\title{
NOTE ON THE REDUCIBILITY OF ALGEBRAS WITHOUT A FINITE BASE*
}

BY M. H. INGRAHAM

It is the purpose of this note to discuss the reducibility of linear associative algebras which are not assumed to possess a finite base. J. H. M. Wedderburn, $\uparrow$ in seeking to generalize certain theorems on the structure of an algebra, has considered algebras in which restrictions are placed upon the character of the idempotent elements. The summations involved in his study need not be finite. This seems to be one natural line of attack.

J. W. Young $\ddagger$ has approached the subject from the point of view of the groups involved. His definition of a finite algebra is, however, unsatisfactory, not being sufficiently restrictive.

I have studied infinite algebras in connection with the results that can be obtained by a use of the "axiom of choice" and the theory of transfinite ordinals. This note, however, does not

* Presented to the Society, December 31, 1928.

$\dagger$ J. H. M. Wedderburn, Algebras which do not possess a finite base, Transactions of this Society, vol. 26 (1924), pp. 395-426.

$¥ \mathrm{~J}$. W. Young, $A$ new formulation for general algebras, Annals of Mathematics, vol. 29 (1927), pp. 47-60. See particularly p. 60 . 
assume the existence of an infinite base for an algebra but merely the usual postulates for a finite linear associative algebra $A$ $=[a]$ over a field $\Xi=[\xi]$, where the postulate requiring the existence of a finite base is replaced by the following extremely mild postulate: for every pair of elements $a_{1}$ and $a_{2}$ in the algebra $A$, we have $1 \cdot a_{1}=a_{1}$ and $a_{1}+a_{2} \cdot 0=a_{1}$, where the elements 1 and 0 are the unit and zero elements respectively of the field $\Xi$ over which the algebra $A$ is taken. There is no difficulty in generalizing the elementary properties of 0 and of a principal unit if it exists.

As in the finite case, a set $S=[s]$ of elements of $A$ is said to be a linear set if for every pair of scalars $\xi_{1}, \xi_{2}$ in $\Xi$ and elements $s_{1}$ and $s_{2}$ in $S, \xi_{1} s_{1}+\xi_{2} s_{2}$ is in $S$. If $C$ is a class of linear sets of elements of $A$, then the sum of these linear sets is defined as the least linear set containing all the linear sets of $C$. This is equivalent to defining the sum as the totality of finite linear combinations with scalar coefficients of the elements of the sets of $C$. The product $S_{1} S_{2}$ of two linear sets $S_{1}$ and $S_{2}$ is the least linear set containing every element of the form $s_{1} s_{2}$, where $s_{1}$ and $s_{2}$ are in $S_{1}$ and $S_{2}$ respectively. I have discussed elsewhere* other properties of linear sets.

As in the case of finite algebras, an invariant proper subalgebra $A_{1}$ of $A$ is one for which both $A_{1} A$ and $A A_{1}$ are contained in $A_{1}$. The sum and intersection of a class of invariant sub-algebras is an invariant sub-algebra (or zero) and the sum of two distinct maximal invariant sub-algebras is $A$. Moreover, we may define the sum of two or more algebras $A_{1}, A_{2}, \cdots$ as a direct sum which we denote $A_{1}(+) A_{2}(+) \cdots$, or $(\Sigma) A_{i}$, if for every $i \neq j, A_{i} A_{j}=A_{j} A_{i}=0$, and the intersection $A_{i} \wedge A_{j}=0$. If $A$ has a principal unit $e$ and $A=A_{1}(+) A_{2}$, then $A_{1}$ has a principal unit $e_{1}$, and $A_{2}$ has a principal unit $e_{2}$, where $e=e_{1}+e_{2}$. We may prove, as in the finite case, the following theorem.

THEOREM 1. If $A_{1}$ and $A_{2}$ are sub-algebras of $A$ either of which has a principal unit, and if $A_{1} A_{2}=A_{2} A_{1}=0$, then $A_{1} \wedge A_{2}=0$ and $A_{1}(+) A_{2}$ is a direct sum.

THEOREM 2. If $A$ has an invariant sub-algebra $A_{1}$ which possesses a principal unit $e_{1}$, then $A$ is reducible and has $A_{1}$ for one component.

* M. H. Ingraham, A general theory of linear sets, Transactions of this Society, vol. 27 (1925), pp. 163-196. 
J. H. M. Wedderburn, in his above mentioned paper, proves this theorem on the basis of a postulate which requires that for every linear set there exists a supplementary set such that the sum of the two is $A$ and the intersection zero. His proof, however, makes no essential use of this hypothesis. The first real difference in this theory from that of finite algebras comes in attempting to generalize the following theorem.*

Any reducible finite algebra $A$ with a principal unit e can be expressed as the direct sum of irreducible algebras each with a principal unit, in one way and only one way apart from the arrangement of the component algebras.

This theorem is not true in the extended theory for there exist algebras of infinite order with principal units which are not expressible as the direct sum of irreducible algebras. Although the existence of a complete reduction of a reducible algebra can not be proved in general, it will be seen as an immediate corollary of Theorem 4 that the uniqueness, except for order of components of any complete reduction into sub-algebras each with a principal unit, does hold in general. This situation is given by Theorems 3 and 4 .

THEOREM 3. If $A$ can be expressed as the direct, sum of sub-algebras in two ways $A=(\Sigma) A_{i}=(\Sigma) B_{i}$, such that $A A_{i}=A_{i} A=A_{i j}$ and $B_{i} A=A B_{i}=B_{i}$, then each $A_{i}$ and $B_{i}$ can be expressed as the direct sum of one or more sub-algebras $A_{i}=(\Sigma) A_{i j}, B_{i}=(\Sigma) B_{i j}$ such that apart from order the $A_{i j}$ 's and the $B_{i j}$ 's are identical.

THEOREM 4. If $A$ has a principal unit, it can not be expressed as the direct sum of more than a finite number of sub-algebras.

Proof of Theorem 3. Let $A_{i j}=B_{j i}$ equal the intersections of $A_{i}$ and $B_{j}$.

$A_{i}=A_{i} A=\Sigma_{j} A_{i} B_{j}$. But $A_{i} B_{j}$ is a portion of the intersection of $A_{i}$ and $B_{j}$, and hence $A_{i}=\Sigma_{j} A_{i j}$ and, moreover, it is a direct sum since $A_{i j}$ is in $B_{j}$ and for every $j \neq k, B_{j} B_{k}=B_{k} B_{j}=0$ and $\left(B_{i} \wedge B_{k}\right)=0$. Similarly, $B_{j}=(\Sigma)_{i} B_{j i}$. Hence $A=(\Sigma)_{i} A_{i}=(\Sigma)_{i j} A_{i}$ $=(\Sigma)_{j}\left(B_{j}\right)=\left(\Sigma_{i j} B_{j i}\right)$, and apart from order, the $A_{i j}$ and the $B_{j i}$ are identical. Moreover, it should be noted that $A_{i j}=A_{i} B_{j}$ $=B_{j} A_{i}$.

* Dickson, Algebras and their Arithmetics, p. 35. 
Corollary. If an algebra $A$ can be reduced to the direct sum of irreducible algebras $A_{i}$, each $A_{i}$ having the property that $A_{i} A=A A_{i}$ $=A_{i}$, this reduction is, apart from order, unique.

It is obvious that $A A_{i}=A_{i} A=A_{i}$ if $A_{i}$ contains a principal unit. Hence reduction of an algebra to irreducible subalgebras each with a principal unit is unique.

In order to prove Theorem 4 , consider $A=(\Sigma) A_{i}$ and containing a principal unit $e$. The principal unit $e$ can be expressed uniquely as a sum of a finite number of elements not more than one of which belongs to any $A_{i}$. Then $e=\sum a_{i}$, where, except for a finite number of values of $i, a_{i}=0$ if $A_{j}$ is such that $a_{j}=0$; then $A_{j}=e A_{j}=0$, and the theorem follows at once.

The following three examples somewhat clarify the situation. Example 1 is an algebra which, although it contains no principal unit, is the direct sum of an infinite number of irreducible subalgebras each with a principal unit and hence, by the corollary to Theorem 4, is uniquely reducible. Example 2 is an algebra with a principal unit containing Example 1 and its invariant sub-algebras as invariant sub-algebras, and hence by Theorem 4 is not reducible to the direct sum of irreducible sub-algebras. Example 3 is the most interesting of the group, being an algebra such that every invariant sub-algebra contains a proper invariant sub-algebra with a principal unit, and hence is reducible. All of these algebras contain denumerable finitely linearly independent bases and hence we do not believe that any simple postulates as to bases for the infinite algebras will yield appreciably greater results in this portion of the theory. Although all the examples are commutative, non-commutative examples can easily be built up as the direct product of these and finite non-commutative division algebras.

ExAMPLE 1. Let $A=$ [all sequences $\left\{\xi_{i}\right\}$ of scalars which are zero except for a finite number of elements]. Let addition, multiplication be the addition and multiplication of corresponding elements, and scalar multiplication the multiplication of each element by the given scalar. Let $A_{i}$ be all the sequences for which $\xi_{j}=0,(i \neq j)$. Then $A=(\Sigma) A_{i}$.

ExAMPLE 2. Let $A=$ [all sequences $\left\{\xi_{i}\right\}$ of scalars such that $\xi_{i}=\xi_{i+1}$ except for a finite set of values for $\left.i\right]$. Let addition, multiplication, and scalar multiplication be defined as above. 
Each $A_{i}$ of Example 1 is an invariant sub-algebra of $A$ but $A \neq$ ( $\Sigma) A_{i}$.

Example 3. Consider $u_{0}=u_{1}+v_{1}$, where

$$
\begin{aligned}
& u_{0}^{2}=u_{0}, \quad u_{1}^{2}=u_{1}, \quad v_{1}^{2}=v_{1}, \\
& u_{0} u_{1}=u_{1} u_{0}=u_{1}, \quad u_{1} v_{1}=0, \quad u_{0} v_{1}=v_{1} u_{0}=v_{1} .
\end{aligned}
$$

In a recursive manner, let

$$
u_{1}=u_{2}+v_{2}, \quad v_{1}=u_{3}+v_{3},
$$

where the table of multiplication for $u_{1}, u_{2}, v_{2}$ can be gotten from that for $u_{0}, u_{1}$ and $v_{1}$ by replacing $u_{0}$ by $u_{1}, u_{1}$ by $u_{2}, v_{1}$ by $v_{2}$, and the multiplication table for $v_{1}, u_{3}$ and $v_{3}$ can be gotten in a similar manner by replacing $u_{0}$ by $v_{1}, u_{1}$ by $u_{3}$, and $v_{1}$ by $v_{3}$. In a similar manner, express each of $u_{2}, v_{2}, u_{3}$ and $v_{3}$ as sums of a pair of elements, etc. If we call $u_{1}$ and $v_{1}$ direct descendents of $u_{0}, u_{2}$ and $v_{2}$ direct descendents of $u_{1}$ and descendents of $u_{0}$, etc., the table of multiplication can be stated in the following geneological fashion. Each $u$ or $v$ is idempotent. An element times its descendent is the descendent; an element times any brother or cousin no matter how far removed is zero. It is also clear that the $u$ 's form a base for the total algebra. Moreover, if any $v$ or $u$ is in an invariant sub-algebra $A_{1}$ of $A$, it and all its descendents form a base for an invariant sub-algebra $A_{2}$ of $A_{1}$ and $A$ of which it is the principal unit. Suppose then $A_{1}$ contains an element $a_{1}=\sum_{i=1}^{n} \xi_{i} u_{i}$. Let $u_{n}$ be a $u$ in the lowest generation represented in $a_{1}$. Then $a_{1} u_{n}=\left(\sum_{1}^{n} \xi_{i}\right) u_{n}$. If $\sum_{1}^{n} \xi_{i} \neq 0, A_{1}$ contains $u_{n}$. If $\sum_{1}^{n} \xi_{i}=0$ and if $w$ is the direct ancestor of $u_{n}$, then $a_{1} w=\sum_{1}^{n-1} \xi_{i} w+$ $\xi_{n} u_{n}=-\xi_{n} v_{n}$ and $A_{1}$ contains $v_{n}$, hence $A_{1}$ contains either $u_{n}$ or $v_{n}$, and hence each of their descendents and hence a proper invariant sub-algebra with a principal unit.

The University of Wisconsin 